\title{
Severe Chronic Venous Insufficiency: Magnitude of the Problem and Consequences
}

\author{
Susan Tsai, MD, Anna Dubovoy, MD, Reid Wainess, BS, Gilbert R. Upchurch, Jr., MD, \\ Thomas W. Wakefield, MD, and Peter K. Henke, MD, Ann Arbor, Michigan
}

\begin{abstract}
The aim of this study was to characterize patients requiring hospitalization for severe chronic venous insufficiency $(\mathrm{CVI})$ at the local and national levels and to analyze factors related to primary amputation. An administrative database (Nationwide Inpatient Sample, 1988-2000) and a single institution (1992-2000) were reviewed using the International Classification of Diseases, 9th ed., Clinical Modification, codes for CVI, excluding phlegmasia and concomitant peripheral vascular occlusive disease codes. Demographics, clinical course, and outcomes were assessed. Descriptive, univariate, and multivariate statistical analyses were used; $p<0.05$ was considered significant. Nationally, CVI occurred with a mean incidence of 92/100,000 admissions, of which $55 \%$ were women, having a mean age of 65 years and a median length of stay of 7 days. Mean hospital charges were $\$ 13,900$ and did not change significantly over time. Acute deep vein thrombosis affected $1.3 \%$, amputation was performed in $1.2 \%$, and in-hospital mortality was $1.6 \%$ The local cohort included 67 patients with a mean age of 51 years; a majority were men (60\%), and $85 \%$ were C6 (of Clinical-Etiologic-Anatomic-Pathophysiology [CEAP]). Patients averaged 23 clinic visits and a median of one hospitalization for CVI care over a 44month follow-up. Twelve patients (18\%) underwent a CVI-related amputation (one transmetatarsal amputation, nine below-knee amputations, and two above-knee amputations). They had fourfold more CVI-related hospitalizations, greater preoperative chronic narcotic use than nonamputee patients ( $85 \%$ vs. $58 \%$ ), but less ongoing wound care needs $(25 \%$ vs. $89 \%)$ (all $p$ values $<0.05)$. However, no significant difference in long-term mortality, number of clinic visits, duration of symptoms, antibiotic courses, or prior venous-related surgeries was found. In those with amputation, ambulatory status was maintained in $75 \%$ at 15 -month follow-up. The physiological and economic costs of severe CVI are significant and have not decreased over more than a decade. Amputation for CVI-related nonhealing wounds has a reasonable outcome. Future therapy must focus on prevention of CVI sequelae.
\end{abstract}

\section{INTRODUCTION}

Venous thromboembolism (VTE) is a common and devastating medical illness that affects hospitalized medical and surgical patients alike. Two primary sequelae result: pulmonary embolism and chronic venous insufficiency (CVI). While good anticoagulant prophylaxis and treatment exist for deep vein

Section of Vascular Surgery, Department of Surgery, University of Michigan, School of Medicine, Ann Arbor, MI.

Presented at the Twenty-sixth Annual Midwest Vascular Surgical Society Meeting, Omaha, NE, September 11, 2004.

Correspondence to: Peter K. Henke, MD, University of Michigan Health System, 1500 East Medical Center Drive, 2210 Taubman Health Care Center, Ann Arbor, MI 48109-0329,E-mail: henke@umich.edu

Ann Vasc Surg 2005; 19: 705-711

DOI: 10.1007/s10016-005-5425-8

(C) Annals of Vascular Surgery Inc

Published online: July 18, 2005 thrombosis (DVT), ${ }^{1}$ a significant percentage of patients develop postphlebitis-related CVI. Less common causes of CVI are primary valvular incompetence and congenital absence of venous valves. ${ }^{2-4}$ Patients affected by this are generally younger and often still working. ${ }^{5-7}$

Severe CVI manifestations of swelling, pain, and ulceration are estimated to occur in $15-40 \%$ of patients with DVT at 8 years. ${ }^{6}$ These manifestations occur more often with postphlebitic syndrome than primary valvular dysfunction. Healing time is variable but often protracted. ${ }^{3}$ A prospective study of 250 patients with venous stasis ulceration (VSU) who were treated with compression demonstrated $57 \%$ and $75 \%$ of ulcers healed at 10 weeks and 16 weeks, respectively. ${ }^{8}$ However, VSU recurrence is common, particularly in patients not compliant with compression therapy; and few series have 
follow-up greater than 24 months. ${ }^{8,9}$ In addition, the socioeconomic impact of VSU is significant. ${ }^{9}$ In the United States alone, direct medical costs for the annual treatment of VSU have been estimated to be between $\$ 150$ million and \$1 billion, with an estimated 2 million lost workdays. ${ }^{3,10}$

The available therapies for CVI manifestations are generally nonsurgical, with exercise, weight loss, elastic compression stockings, and wound care. ${ }^{4,8,11,12}$ While superficial venous ablation has shown promise in a prospective, randomized trial, ${ }^{13}$ deep system insufficiency reconstructions have variable results outside of specialized centers and few have been subjected to randomized prospective evaluation. ${ }^{14,15}$ Although VSU rarely necessitates amputation, patient debilitation and discomfort can be significant. Patients with severe CVI manifestations (C5-6 of the Clinical-EtiologicAnatomic-Pathophysiology [CEAP] classification ${ }^{16}$ ) frequently report a reduction in quality of life: $81 \%$ of patients experience decreased mobility and 57\% report severe limitation in mobility. ${ }^{7,12}$

Because many of these patients require numerous clinical visits as well as occasional hospitalizations, the goal of this study was to evaluate the current national epidemiology, incidence, and cost of CVI and then to review a local series of patients who were admitted with CVI-related wound problems and characterize their clinical course outcomes, including those who underwent primary amputation.

\section{METHODS}

This study utilized two databases. The first is the Nationwide Inpatient Sample (NIS), an administrative database that is a $20 \%$ stratified random sample of all hospital discharges in the United States and includes years 1988-2000. The database is maintained by the Agency for Health Care Research and Quality as part of the Health Care Cost and Utilization Project. ${ }^{17}$ Patients were identified by the International Classification of Diseases, 9th ed., Clinical Modification ${ }^{18}$ (ICD-9CM), hospital primary discharge codes for CVI-related diagnoses: postthrombotic syndrome, 459.1; venous insufficiency, 459.81; VSU, 454.0; and deep vein phlebitis, 451.11. Coexistence of acute DVT was defined by ICD-9CM code 481.1. Patients in this group who had concomitant codes for peripheral vascular occlusive disease (PVOD; $440.21,440.22,440.23,440.24,440.29)$ and phlegmasia (451.19) were excluded to increase the specificity for venous-related disease out- comes. Procedural codes for amputation below the knee (BKA) or above the knee (AKA) (84.10, $84.15,84.17$ ) were denoted for the same hospitalization as the index CVI-coded patients. Outcome measures evaluated were amputation rate, length of stay (LOS), mortality, and inflation-adjusted cost of hospitalization.

The local series included consecutive patients over a period of 8 years (1992-2000) using the same diagnostic codes to isolate these hospitalized inpatients. Only patients who were hospitalized at least once for their CVI-related wound problems were included. All patients had a VSU at some point as the cause for index admission and were cared for by the Vascular Surgery Service at the University of Michigan. The size and locations of the various ulcers were not determined as they varied significantly over time. A majority of the patients had CVI diagnosis confirmed with either ascending/ descending venography or air plethysmography, using standard positive criteria. ${ }^{19}$ During the time frame analyzed, duplex diagnosis of venous insufficiency was not routinely used. Patients with a history of phlegmasia were excluded, as were those with interventions (e.g., surgical bypass or endovascular) performed for PVOD. A detailed review of the patients' electronic medical charts identified demographic data including age, gender, anticoagulation status, CEAP classification (based on clinic and discharge summary descriptors), and duration of symptoms. Medical resource utilization data, including clinic visits, number of courses of antibiotics, narcotic pain medication usage, duration of outpatient follow-up for CVI care, number of clinic visits and hospital admissions, and need for venousrelated surgery. CVI-related surgeries included varicosity excision, split thickness skin grafting (STSG) to the ulcer bed, subfascial endoscopic perforator surgery (SEPS), venous valve reconstruction, and venous bypass procedures. For those patients who were lost to follow-up or deceased, the ambulatory status was abstracted from the last clinic documentation.

Statistical analysis utilized the Mann-Whitney U or Student $t$-test for continuous variables and the chi-squared or Fisher exact test for univariate analysis. Logistical regression with dichotomous variables was used to determine independence of associated factors with specific outcomes of mortality and amputation. Evaluation of the local data with smaller patient numbers utilized univariate analysis only. SPSS (Chicago, IL) statistical software was used. $p<0.05$ was deemed significant. The study was approved by the University of Michigan Institutional Review Board. 

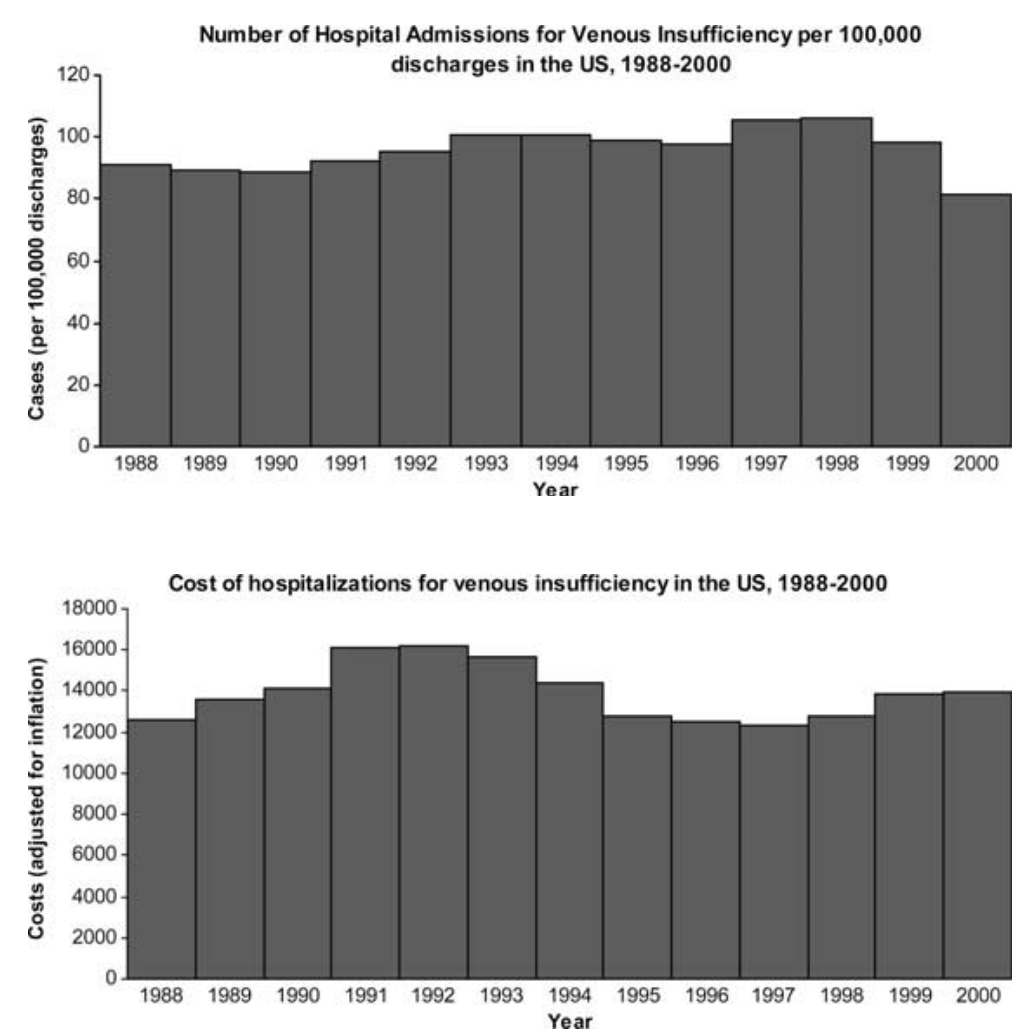

Fig. 1. Hospital admissions (cases) for primary diagnostic coded CVI-related discharges per 100,000 patient discharges 1988-2000.

Fig. 2. Inflation-adjusted hospital care cost (in dollars) for CVI-related discharge codes 1988-2000.
Table I. National cohort demographics

\begin{tabular}{ll}
\hline Number & 80,857 \\
Age (years) & $65 \pm 18$ \\
Gender & $55 \%$ men, $45 \%$ women \\
Concurrent VTE & $1.3 \%$ \\
BKA & $1.1 \%$ \\
AKA & $0.1 \%$ \\
Mortality & $1.6 \%$ \\
LOS (days, median) & 7 \\
\hline
\end{tabular}

\section{RESULTS}

Nationally, from 1988 to 2000, CVI-related diagnoses accounted for a mean of 92 per 100,000 patient admissions without significant change over time (Fig. 1). In this cohort, 55\% were women, with a mean age of 65 years and a median LOS of 7 days (range 1-143 days). Mean inflation-adjusted hospital charges were $\$ 13,900$, with little variation over the time period studied (Fig. 2). Acute DVT was a codiagnosis in $1.3 \%$, and amputation was performed in $1.1 \%$. In-hospital mortality was $1.6 \%$ (Table I). Logistical regression was done to determine independent factors associated with mortality, BKA, and AKA (Table II). While administrative databases are limited in the specificity of patient factors that can be analyzed, mortality was primarily determined by age, emergent or urgent admission, associated medical diseases, as well as need for BKA and AKA. BKA $(n=889)$ was associated with elective admission category (e.g., urgent and emergent admission type was associated with lower risk of amputation) as well as older age. Female gender was protective, but no significant association with diabetes, renal failure, coexistence of an acute DVT, or race was found. A similar predictive distribution of associated factors was shown with AKA (data not shown), though the number of patients having this procedure associated with CVI was very low $(n=80)$.

At the local level, 67 patients were included, with a mean age of 51 years; $60 \%$ were men (Table III). Most patients had active VSU, with $85 \%$ having C6 classification, and averaged 23 clinic visits for their CVI care. The median number of hospitalizations was one over a 44-month mean follow-up. Of this group, 12 patients underwent a CVI-related amputation: one transmetatarsal amputation (TMA), nine BKA, and two AKA. All in the amputee group were C6 class and had fourfold more hospitalizations, and a greater proportion used narcotics chronically for pain than the nonamputee group (all $p<0.05$ ). Not surprisingly, fewer patients required ongoing wound care after 
Table II. Logistic regression

\begin{tabular}{lllcr}
\hline & Factor & Odds ratio & 95\% Confidence interval & $p$ \\
\hline Mortality & Age > 55 years & 3.1 & $2.1-4.4$ & $<0.001$ \\
& Admission type & & & $<0.001$ \\
& $\quad$ Urgent & 1.48 & $1.21-1.82$ & $<0.001$ \\
& Emergent & 2.1 & $1.7-2.5$ & $<0.001$ \\
& BKA & 3.8 & $2.5-5.9$ & $<0.001$ \\
AKA & 5.1 & $3.4-7.4$ & $<0.001$ \\
& Renal failure & 4.5 & $2.7-7.6$ & 0.003 \\
BKA & Age $>$ 55 years & 1.67 & $1.2-2.2$ & $<0.001$ \\
& Female & 0.50 & $0.41-0.62$ & $<0.001$ \\
& Admission type & & & $<0.001$ \\
\hline & Urgent & 0.55 & $0.42-0.72$ & $0.53-0.85$ \\
\hline
\end{tabular}

Nonsignificant associations for mortality included gender $(p=0.26)$, race, VTE, and diabetes. Nonsignificant associations for BKA included presence of diabetes, renal failure, VTE, and race.

Table III. Local series

\begin{tabular}{|c|c|c|c|c|}
\hline Patient characteristics $(n=67)$ & Total & Nonamputees & Amputees & $p$ \\
\hline Age (years) & $51 \pm 2$ & $50 \pm 4.4$ & $57.3 \pm 2.3$ & NS \\
\hline Gender & 41 men, 27 women & 32 men, 23 women & 9 men, 4 women & NS \\
\hline C6 class $(\%)$ & $56(85 \%)$ & $45(81 \%)$ & $11(100 \%)$ & NS \\
\hline Reflux + obstruction (\%) & $25(37 \%)$ & $20(36 \%)$ & $5(42 \%)$ & NS \\
\hline Number of clinical visits & $23 \pm 3$ & $21 \pm 2.8$ & $31.27 \pm 10$ & NS \\
\hline Duration of CVI symptoms (months) & $45 \pm 4$ & $43.9 \pm 29.8$ & $51 \pm 8$ & NS \\
\hline Hospitalizations (median) & 1.0 & 1.0 & 4.0 & $<0.001$ \\
\hline Venous surgeries & $59(88 \%)$ & $35(63 \%)$ & $8(67 \%)$ & NS \\
\hline Mean number of antibiotic courses & $2.7 \pm 0.5$ & $2.6 \pm 0.8$ & $3.3 \pm 0.4$ & NS \\
\hline Chronic patient narcotic use (\%) & $43(64 \%)$ & $32(74 \%)$ & $11(85 \%)$ & 0.04 \\
\hline Compression stockings (\%) & $48(72 \%)$ & $44(75 \%)$ & $4(33 \%)$ & NS \\
\hline Ongoing wound care (\%) & $52(78 \%)$ & $49(89 \%)$ & $3(25 \%)$ & $<0.001$ \\
\hline Warfarin (Coumadin, \%) & $33(49 \%)$ & $28(49 \%)$ & $5(38 \%)$ & NS \\
\hline Ambulatory & $54(90 \%)$ & $55(100 \%)$ & $9(75 \%)$ & NS \\
\hline Mortality & $12(19 \%)$ & $8(15 \%)$ & $4(33 \%)$ & NS \\
\hline Follow-up (months) & $44 \pm 4$ & $45 \pm 4$ & $34 \pm 8$ & NS \\
\hline
\end{tabular}

amputation compared with nonamputees. Importantly, those patients undergoing an amputation were unlikely to have significant PVOD as the affected limb mean ankle brachial index was 0.93 $(n=5)$ or no pulse deficits were noted in the chart. One patient had a perioperative flap infection that required flap revision, but salvage of the BKA was maintained.

No significant difference in long-term mortality, number of clinic visits, antibiotic courses, duration of symptoms, or prior venous-related surgeries was found. Specifically, the types of venous-related surgery in the amputee group included varicose excision $(n=1,7 \%)$, SEPS $(n=1,7 \%)$, STSG $(n=2,14 \%)$, valve reconstruction $(n=4,31 \%)$, and veno-veno bypass $(n=1,7 \%)$; while the nonamputee group had a similar distribution: varicosity excision $(n=13,23 \%)$, SEPS $(n=7,13 \%)$, STSG $(n=15,27 \%)$, valve reconstruction $(n=8$, $15 \%)$, and veno-veno bypass $(n=8,15 \%)$. Longterm mortality was $19 \%$ for the entire group at 44 month follow-up and was not significantly different between amputees and nonamputees $(p=0.24)$.

\section{DISCUSSION}

The current report substantiates other reports in that CVI with resultant limb swelling, pain, and ulceration is a common disease that incurs significant morbidity and cost. ${ }^{3,5,7,12,20,21}$ The epidemiological data reinforce both the significant economic 
impact and patient morbidity associated with severe CVI (e.g., C6 classification). Discouragingly, the current study showed that neither inflationadjusted mean hospital charge nor LOS decreased over the last decade. This may reflect little advancement in the basic therapy for severe CVI. At this time, advanced VSU requires an intense regimen of wound care, compression, chemical and mechanical debridement, and antibiotics if necessary. The national data do not include the costs associated with outpatient CVI care, the setting in which most patients receive care, and hence underestimates the true overall costs. In one series, the mean cost for outpatient wound care over a 90day period approached $\$ 6,000 .^{22}$ Another study reported outpatient treatment costs of VSU care ranging $\$ 1,444-2,711$ over a 10 -week period. ${ }^{20}$ While a large number of clinic visits was documented, the burden for the patient and family in travel time, loss of work hours, and ongoing home care was not quantified. Similarly, the cost and physiological impairment related to chronic use of narcotics and antibiotics can also only be speculated.

While the national amputation rate of $1.2 \%$ is likely accurate, it may overestimate the true number who had this for CVI-related problems due to limited specificity in identifying patients by ICD9CM coding. Despite efforts to exclude patients with coexistent arterial disease, this may not have completely eliminated other disease processes, such as severe infection, that may have resulted in an amputation. However, urgent or emergent admission was actually associated with lower amputation risk in this group, suggesting a planned admission for these amputation procedures. That is, we speculate that patients who underwent a CVI-related amputation were electively admitted for such and that this was not done routinely in sicker patients. Similarly, the fact that diabetes mellitus and renal failure were not associated with the evaluated outcomes suggests that this patient population is different from most arterial vascular disease patients who undergo amputation. While mortality was increased in the national series among those undergoing amputation, this was likely due to underlying associated diseases or a generally debilitated condition not able to be objectified by the limitations of an administrative data $\operatorname{set}^{23}$ and was quite low at $\sim 1 \%$.

At the local level, our series was small and lacked the power to discern differences in patient disease parameters that would predispose toward ultimately requiring an amputation. While the overall amputation rate was $18 \%$, significantly higher than the national data, this represents a very select series with all end-stage tertiary referral patients. Further, our group consisted of patients admitted solely for CVI-related wound complications and/or operations related to this. The national data set was broader in inclusivity. This tenet is supported by the fact that all amputees were C6 class, most having nearly a decade of CVI-related symptoms and ulceration, a significant number of hospitalizations, and requiring chronic narcotics for pain control as well as numerous prior venous reconstructive surgeries. Unlike patients who have amputations secondary to arterial disease, these patients seem to have reasonable longevity, rehabilitation potential, and likely better use of a prosthesis than those who have arterial disease, where death and disability rates are significant. ${ }^{24}$ For example, no patient in this series required a higherlevel amputation, in contrast to many with amputations for arterial disease. Approximately three-quarters of amputated venous patients were ambulatory, in contrast to an average rate of 30$40 \%$ at our hospital (unpublished observations).

VSU often occurs as a sequela of previous DVT. Numerous investigators have found higher rates of ulcer recurrence in patients without compression therapy, and better results are often obtained in controlled, intensive trials. This study did not directly assess VSU healing, though the fact that most had ongoing clinic visits and hospitalizations reflects the relapsing nature of VSU. While those with significant PVOD requiring an intervention were excluded from the local cohort, patients with mild PVOD were not; and this may also have contributed to the need for amputation, though the mean ankle brachial index was nearly normal. However, not all patients in this series had this objectively determined preoperatively. The presence of mild arterial disease coexistent lymphedema and diabetic foot problems may contribute to poor wound healing. ${ }^{10}$ Further investigations into the effect of mild arterial insufficiency as well as diabetes, coexistent VTE, and hemodialysis-dependent renal failure on VSU healing may be warranted to better prognosticate who might benefit from an amputation, though none of these was predictive from the NIS data. Similarly, because of the recurring nature, severity, and location of the VSUs in this series, size was not evaluated in the current study but is known to correlate with healing. ${ }^{25}$

While DVT treatment with anticoagulation effectively treats acute thrombus, a significant percentage of patients go on to develop postphlebitic syndrome and CVI. 4,6,11,26 More intensive efforts should be made in the prevention of DVT by 
vascular surgeons, as well as early preventive education of CVI by nonvascular surgeons in particular. Whether rapid and complete DVT thrombolysis via a catheter-directed approach compared with standard anticoagulation is more efficacious has yet to be determined. ${ }^{27}$ However, early experience with this technique suggests fewer longterm CVI sequlae. ${ }^{28}$ Currently, early ambulation and compression stocking therapy after acute DVT and rapid achievement of therapeutic heparin anticoagulation should decrease the incidence of later severe CVI manifestations. ${ }^{29}$ For example, institution of stocking compression (20-40 mm Hg graded) started within 2-3 weeks after acute DVT reduced subsequent severe CVI by $50 \% .^{11}$ This measure has also been shown to be cost-saving but has yet to be fully reimbursed. ${ }^{30}$

The limitations of this study are several. First, the NIS database lacks a certain specificity with relation to patient disease and outcome. While we used the primary discharge diagnosis codes of venous insufficiency and limited this to patients who did not have concurrent PVOD codes to remove patients who might have had an amputation for another reason, this is not totally specific. Furthermore, whereas the data are quite accurate with regard to certain demographics, mortality, and LOS, the predictors for patient-specific outcomes are more variable as the compilation of these data depends on trained coders rather than physicians or nurse abstractors. Second, the local data and the operative decision to amputate were surgeon-specific, and no evidence-based protocols were used. Some amputee patients had mild PVOD and diabetic wound problems that likely contributed to the decision, but inclusion into this cohort was based firmly on review of the primary amputation indication in the medical record. There is a lack of literature supporting or refuting amputation for CVI sequelae with the exception of phlegmasia cerulea or phlegmasia alba dolens when gangrene is present, and these patients were specifically excluded in the current series. Thus, the recommendations from this series may not be applicable to all institutions.

In conclusion, severe CVI is a costly and morbid disease that, because of its chronicity and rarely life-threatening prognosis, receives little national attention. In highly selected patients who have failed venous reconstructive surgeries, amputation may be reasonable therapy for chronic VSU and/or intractable associated pain. These sobering data with regard to cost, magnitude of clinical and hospitalization utilization, and amputation rate lend further support to the recommendations that better prophylactic anticoag- ulant protocols be instituted in hospitalized patients to prevent DVT from occurring and that rapid evidence-based therapy be applied for those with established DVT.

\section{REFERENCES}

1. Goldhaber SZ, Tapson VF. A prospective registry of 5,451 patients with ultrasound-confirmed deep vein thrombosis. Am J Cardiol 2004;93:259-262.

2. Heit JA, Silverstein MD, Mohr DN, et al. The epidemiology of venous thromboembolism in the community. Thromb Haemost 2001;86:452-463.

3. Heit JA, Rooke TW, Silverstein MD, et al. Trends in the incidence of venous stasis syndrome and venous ulcer: a 25 year population-based study. J Vasc Surg 2001;33:10221027.

4. Nicolaides AN., Nicolaides AN. Investigation of chronic venous insufficiency: a consensus statement (France, March 59, 1997). Circulation 2000;102:E126-E163.

5. Ruckley CV. Socioeconomic impact of chronic venous insufficiency and leg ulcers. Angiology 1997;48:67-69.

6. Prandoni $\mathrm{P}$, Lensing AW, Cogo A, et al.The long-term clinical course of acute deep venous thrombosis. Ann Intern Med 1996;125:1-7.

7. Delis KT, Bountouroglou D, Mansfield AO. Venous claudication in iliofemoral thrombosis: long-term effects on venous hemodynamics, clinical status, and quality of life. Ann Surg 2004;239:118-126.

8. Marston WA, Carlin RE, Passman MA, Farber MA, Keagy BA. Healing rates and cost efficacy of outpatient compression treatment for leg ulcers associated with venous insufficiency. J Vasc Surg 1999;30:491-498.

9. Phillips T, Stanton B, Provan A, Lew R. A study of the impact of leg ulcers on quality of life: financial, social, and psychologic implications. J Am Acad Dermatol 1994;31:49-53.

10. Baker SR, Stacey MC, Jopp-McKay AG, Hoskin SE Thompson PJ. Epidemiology of chronic venous ulcers. Br J Surg 1991;78:864-867.

11. Brandjes DP, Buller HR, Heijboer $\mathrm{H}$, et al. Randomised trial of effect of compression stockings in patients with symptomatic proximal-vein thrombosis. Lancet 1997;349:759762 .

12. Nicolaides AN. Edema in chronic venous insufficiency and the effect of modern pharmacotherapy. Angiology 2000;51:1-2.

13. Barwell JR, Davies CE, Deacon J, et al. Comparison of surgery and compression with compression alone in chronic venous ulceration (ESCHAR study): randomised controlled trial. Lancet 2004;363:1854-1859.

14. Kistner RL, Eklof B, Masuda EM. Deep venous valve reconstruction. Cardiovasc Surg 1995;3:129-140.

15. O'Donnell TFJ. Venous valve transplantation and vein transposition for valvular incompetence of deep veins In: Gloviczki, P, Yao, JST, eds. Handbook of Venous Disorders. 2nd ed. London: Arnold, 2001, pp 336-345.

16. Porter JM, Moneta GL. Reporting standards in venous disease: an update. International Consensus Committee on Chronic Venous Disease. J Vasc Surg 1995;21:635-645.

17. Agency for Healthcare Research and Quality. Healthcare Cost and Utilization Project (HCUP-9). Nationwide Inpatient Sample, Release 9. 2000.

18. Public Health Service. International Classification of Diseases, 9th rev. Clinical Modification. Washington, DC: U.S. Department of Health and Human Services, 1988. 
19. Marston WA. PPG, APG, duplex: which noninvasive tests are most appropriate for the management of patients with chronic venous insufficiency? Semin Vasc Surg 2002;15:13-20.

20. O'Donnell TF, Jr, Browse NL, Burnand KG, Thomas ML. The socioeconomic effects of an iliofemoral venous thrombosis. J Surg Res 1977;22:483-488.

21. Vanhoutte PM, Corcaud S, de Montrion C. Venous disease: from pathophysiology to quality of life. Angiology 1997;48: 559-567.

22. Olin JW, Beusterien KM, Childs MB, Seavey C, McHugh L, Griffiths RI. Medical costs of treating venous stasis ulcers: evidence from a retrospective cohort study. Vasc Med 1999;4:1-7.

23. Birkmeyer JD. Using administrative data for clinical research. In Souba W, Wilmore D, eds. Surgical Research. Academic Press, 2001.

24. Krupski WC. Overview of extremity amputations. In Rutherford R, ed. Vascular Surgery. Philadelphia: WB Saunders, 2000.
25. Phillips TJ, Machado F, Trout R, Porter J, Olin J, Falanga V. Prognostic indicators in venous ulcers. J Am Acad Dermatol 2000;43:627-630.

26. O'Shaughnessy AM, Fitzgerald DE. Underlying factors influencing the development of the post-thrombotic limb. J Vasc Surg 2001;34:247-253.

27. Bates SM, Ginsberg JS.. Clinical practice. Treatment of deep-vein thrombosis. N Engl J Med 2004;351:268277.

28. Comerota AJ. Quality-of-life improvement using thrombolytic therapy for iliofemoral deep venous thrombosis. Rev Cardiovasc Med 2002;3(Suppl):S61-S67.

29. Prandoni P. Toward the simplification of antithrombotic treatment of venous thromboembolism. Ann Intern Med 2004; 140:925-926.

30. Korn P, Patel ST, Heller JA, et al. Why insurers should reimburse for compression stockings in patients with chronic venous stasis. J Vasc Surg 2002;35:950957. 